XXXIX.

\title{
FURTHER OBSERVATIONS ON THE RADICAL TREATMENT OF PERITONSILLAR ABSCESS.
}

\author{
By Clement F. Theisen, M. D.,
}

\section{Albany.}

In a paper giving the report of an epidemic of acute infection of the throat with abscess formation (Trans. A. L. A., 1916), the writer reported forty-four cases of peritonsillar abscess occurring in that epidemic. In fourteen of these cases, after a dissection backward toward the capsule of the tonsil, to locate the pus, the upper lobe, which had been loosened by the dissection, was removed. This leaves a large opening for drainage, and the cases so operated upon cleared up much more quickly than when simply the usual incision was made.

Ballenger, I believe, was one of the first to suggest a dissection backward toward the capsule in cases of peritonsillar abscess. The pus is present very soon after the attack begins in the majority of the cases, and we often fail to reach it early in the case, because we do not cut deeply enough toward the capsule. Atypical cases with very little pus and great swelling, edema and inflammation of the surrounding parts, presenting the clinical picture of a typical peritonsillar infection, occur, but they are fairly exceptional cases. In the vast majority of the cases the pus is present, but sometimes difficult to locate when it is confined between the body of the tonsil and the superior constrictor muscle. It is in this class of cases, particularly, that Barnes, in his paper on the radical treatment of peritonsillar abscess, recommends dissecting out the tonsil early during the acute stage. He has reported excellent results in a number of cases.

We frequently see cases so late that, owing to the great edema and inability of the patient to separate the teeth, it is difficult to find the usual anatomic landmarks.

In this class of cases the writer has not attempted the removal of any part of the tonsil during the acute stage. When the infection has gone so far as this, the pus is reached with- 
out difficulty almost anywhere in the peritonsillar region.

Since the paper above referred to was published the writer has used the method described at that time-i. e., the removal of part of the tonsil after reaching the pus, in twenty cases, and the complete removal in sixteen. These were all suitable cases, seen early, before there was much swelling and difficulty in opening the mouth. The majority of the cases occurred in adults, and this is, of course, the rule in most peritonsillar abscesses. They do occur, however, and not so very infrequently, in children.

In the series of cases reported in my paper in 1916, fortyfour in all, four occurred in young children.

Five of the thirty-six cases, reported in the paper read today, occurred in children, and were operated upon under ethyl chloride anesthesia. They were all very nervous children, and could not have been easily managed under local anesthesia. All the adult cases were operated upon with cocain and adrenalin. When possible, I think it is safer to use local anesthetics in these cases, particularly when there is considerable edema filling up part of the throat. I have found, and that is probably the experience of most of us, that as soon as the mouth gag is inserted, after the patient is practically under the anesthetic, he begins to act badly. The same is true to even a greater extent of cases of retropharyngeal abscess in young children.

Barnes, in the paper before referred to (Trans. A. L. A., 1915), speaks of the danger of pulmonary infection from inspired pus, when a general anesthetic is used. He has performed a complete tonsillectomy in his cases on about the third day, and has had no complications of this kind. His cases all got along very well indeed, with no more distress than when the tonsil is removed under normal conditions. He has not found, when care is taken in dissecting out the tonsil with as little bleeding as possible, that there was any spread of the infection. The possibility of the aspiration of pus is of course largely done away with when local anesthetics are used.

The operation the writer has performed in selected cases, when the edema of the surrounding parts is not too great, is as before stated, a capsular dissection of the tonsil backward 
until the pus is reached, and then the immediate removal of the part of the tonsil that had been loosened by the dissection.

There is no reason why the entire tonsil should not be taken out at the time, and this was done in my last sixteen cases. There is surprisingly little discomfort after the operation, and the relief following the much more thorough drainage than is afforded by the ordinary incisions is much greater and more prompt. I have not found that this operation, performed during the acute stage, is attended by any increased risk of a spread of the infection, particularly if care is taken to perform the operation with as little bleeding as possible.

The ordinary incision is often attended with much more bleeding and cannot be as readily controlled. When we bear in mind that in a peritonsillar abscess we are really dealing with an infection which soon involves the entire sinus tonsillaris, the risk of causing a more extensive septic process by our operative procedures need not be considered very seriously. Even if this were not so, the great relief following the operation so promptly is well worth the slight risk.

The ordinary incision, unless it is very free, closes very easily, and has to be kept open by packing or by stretching with a forceps every day. Patients as a rule object to this very much more than to the more radical operations, at least that has been the writer's experience. The abscess cavity, unless it is very superficial, drains badly after the ordinary incision, and not infrequently further abscesses form after the patient is apparently well. A tonsil is never perfectly normal after a peritonsillar infection, and in many cases the socalled "quinsy habit" is established, with an attack once or twice a year. The tonsils must eventually come out, and their removal, in selected cases, as early as possible during the acute attack is a rational operation.

I will not report any individual cases, but would like briefly to report a case that really brought home to me the necessity of some more radical operation, in peritonsillar infections, than the simple incision, and started the method of treatment I have used since that time.

A young woman, aged twenty-five years, developed what appeared to be a slight tonsillitis, not follicular, with very moderate inflammation of particularly one tonsil. I gave her 
an astringent gargle, told her to use ice, and did not expect to see her again. On the following day she appeared at my office again, stating that she had great pain in swallowing and was feeling much worse generally. The inflammatory process was now confined to the left tonsil, with very little swelling, nothing to suggest a peritonsillar infection. Her distress was so out of proportion to the findings that I decided to make an incision in the usual place. I did not strike any pus. This was repeated every day, punctures and incisions being made in different places, for four or five days, and no pus was found. The patient was getting more discouraged all the time, and I did not blame her. She could swallow nothing but liquids, with great difficulty. During all this time the swelling increased only very slightly, and clinically the condition could not have been called at all typical of a quinsy.

I was about on the point of advising the patient to get another doctor, when it occurred to me that she probably had a deep collection of pus, a very small abscess cavity. I then grasped the tonsil with a forceps and carried my incision directly back toward the capsule, and entered a very small abscess cavity containing very little pus. The dissection was completed and the upper half of the tonsil removed, and in a few days we were both happy.

I suppose we have all had this same experience many times, and do not reach the pus the first time simply because we do not go back far enough toward the capsule. I am speaking now only of atypical cases, such as the one reported, and cases in which we fail to reach the pus during the first few days after the onset of the infection.

\section{CONCLUSIONS.}

The radical method of treatment for the quick relief of the distressing symptoms was used in thirty-six selected cases, in twenty of which the part of the tonsil that had been loosened by the dissection in reaching the pus was removed at the time. In sixteen cases a complete tonsillectomy was immediately performed after the pus was evacuated.

The operation is performed as early as possible, usually within two or three days after the onset of the attack, and in selected cases. In cases in which there is so much edema of 
the parts that the anatomic landmarks cannot be easily determined the radical method was not attempted.

In this type of cases the inability to separate the teeth is usually so great that the radical operation would be very difficult. Local anesthesia, because of the possibility of the aspiration of pus under general anesthesia, is always used except in young children.

The risk of a spread of the infection is so slight, and has never been experienced by the writer, that there is no reason why a complete tonsillectomy should not be performed in every suitable case, at the time the abscess cavity is opened.

The cases reported in this paper were all unilateral abscesses, but the writer has used the same method in bilateral cases, which are fortunately not as common.

No unfavorable symptoms occurred when both tonsils were operated upon. As a matter of fact, the pain in swallowing after the operation does not last much longer, and the case runs about the same postoperative course as when the tonsils are removed under normal conditions. There is at times some temperature, particularly in children, and the tonsillar fossæ get back to normal conditions rather slowly. 